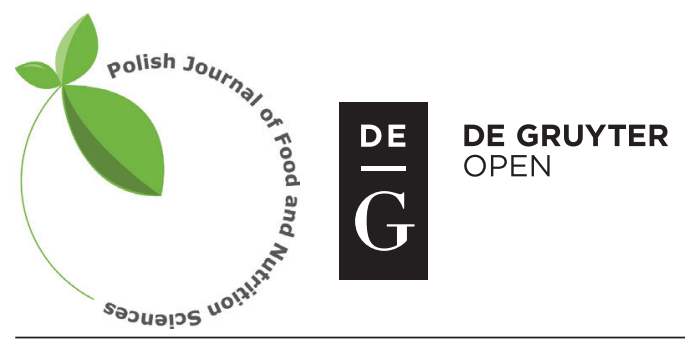

Pol. J. Food Nutr. Sci., 2016, Vol. 66, No. 3, pp. 167-171 DOI: $10.1515 /$ pjfns-2015-0036

Original article

Section: Food Quality and Functionality

\title{
Antioxidant Capacity of a Turkish Traditional Alcoholic Drink, Raki
}

\author{
Gorkem Yalcin* \\ Department of Analytical Chemistry, Faculty of Pharmacy, Ege University, 35100, Bornova, Izmir, Turkey
}

Key words: raki, beverage, spirit, antioxidant capacity

Raki is an aniseed flavoured traditional Turkish alcoholic drink. Antioxidant capacity of raki samples from different commercial brands were evaluated by CUPRAC, DPPH, TEAC and ORAC assays and correlations between these assays and total phenolic content were also investigated. Additionally, the one-way ANOVA and Bonferroni tests were performed to compare differences between values of the samples. Results indicated that different raki samples exhibited different antioxidant capacity and total phenolic content. The mean antioxidant capacity values of samples were in the order of: ORAC $>$ TEAC $>$ CUPRAC $>$ DPPH. The correlations of total phenolic content of samples with their CUPRAC, TEAC and ORAC results were found statistically significant, while DPPH assay showed no significant correlation.

\section{INTRODUCTION}

Raki is an aniseed flavoured distilled spirit that is widely consumed in Turkey. It is often served with seafoods or meze. Raki is described as a spirit that is produced by double distillation of suma or suma mixed with agricultural based ethanol and flavouring it with aniseed (Pimpinella anisum) in the Turkish Food Codex [Anli \& Bayram, 2010]. The main raw material of raki is suma, which is a distillate with a maximum $94.5 \%$ ethanol content. Raisins, molasses and/or grape must are used for suma production [Yucesoy \& Ozen, 2013]. In the raki production process, traditional copper alembics with a maximum capacity of $5000 \mathrm{~L}$ are used for distillation of suma. After the distillation, distillate is diluted to $45 \%$ alcohol. Finally, sugar is added to end product in order to sweeten raki and it is stored for ageing at least one month before bottling [Anli \& Bayram, 2010].

Many in vitro assays have been conducted to evaluate the antioxidant capacity of food products and drinks [Pellegrini et al., 2003; Li et al., 2005; Zulueta et al., 2007, 2009a; Schwarz et al., 2009]. However, determination of antioxidant capacity of a particular sample cannot be performed accurately by any single assay [Ozyurek et al., 2011; Bernaert et al., 2012]. Consequently, at least two assays should be used in order to assess antioxidant capacity accurately [Li et al., 2011; Meng et al., 2011].

Plants are an important source of natural antioxidants. It was reported that aniseed had strong antioxidant activity [Gülçın et al., 2003]. Fu et al. [2011] demonstrated antioxidant capacity of grape samples. The use of grapes and/or

\footnotetext{
* Corresponding Author: Tel.: +90 232 3113992; Fax: +90 232 3885258;

E-mail: gorkemy1@gmail.com, gorkem.yalcin@ege.edu.tr
}

grape based material, aniseed to produce raki may contribute to the ingestion of naturally occurring antioxidant compounds. However, the antioxidant properties of raki have not been reported elsewhere. Therefore, antioxidant capacity of raki samples from different commercial brands were analysed in this research and four different assays were tested for antioxidant capacity determination to provide a more reliable investigation. In addition, total phenolic content was also analysed to evaluate correlations between total phenolic content and antioxidant capacity.

\section{MATERIAL AND METHODS}

\section{Samples}

Seven different commonly consumed raki samples of different brands were purchased from local supermarkets and stored in their original bottles at $4^{\circ} \mathrm{C}$. Samples were classified and coded according to their raw materials used in the production: Raki samples produced from dried type grapes (A, B, E, F), fresh type grapes (C, G) and fresh-dried type grapes (D, which is a combination of fresh and dried type grapes).

\section{Antioxidant capacity assays}

The DPPH (2,2-diphenyl-1-picrylhydrazyl) radical scavenging capacity was determined using the method described by Tafulo et al. [2010] with some modifications. Added amount of sample and DPPH reagent, reaction time modifications were applied to the method. Briefly, $0.25 \mathrm{~mL}$ of samples were mixed with $2.75 \mathrm{~mL}$ of $0.1 \mathrm{mmol} / \mathrm{L}$ DPPH. The absorbance at $517 \mathrm{~nm}$ was determined after reaction time of $30 \mathrm{~min}$. The standard curve was constructed using Trolox $(0.02-0.10 \mathrm{mmol} / \mathrm{L})$ and the results were expressed as $\mu \mathrm{mol} / \mathrm{L}$ Trolox equivalent (TE). 
CUPRAC (cupric reducing antioxidant capacity) assay was carried out by using method described by Apak et al. [2008] with slight modification. Added amounts of reagents were modified in the method. In brief, $0.75 \mathrm{~mL}$ copper (II) chloride $(10 \mathrm{mmol} / \mathrm{L}), 0.75 \mathrm{~mL}$ neocuproine $(7.5 \mathrm{mmol} / \mathrm{L})$, $0.75 \mathrm{~mL}$ ammonium acetate buffer $(1 \mathrm{~mol} / \mathrm{L}, \mathrm{pH}=7.0)$, $0.75 \mathrm{~mL}$ water and $0.1 \mathrm{~mL}$ sample were mixed in a cell. After $30 \mathrm{~min}$, the absorbance at $450 \mathrm{~nm}$ was measured. Trolox $(0.05-0.50 \mathrm{mmol} / \mathrm{L})$ was used as a reference standard. Results were expressed as $\mu \mathrm{mol} / \mathrm{L} \mathrm{TE}$.

For TEAC (trolox equivalent antioxidant capacity) assay, method described by Re et al. [1999] was used with some modifications. Added amount of sample and ABTS reagent, reaction time modifications were applied to the method. ABTS radical cation $\left(\mathrm{ABTS}^{\cdot+}\right)$ stock solution was prepared from $7 \mathrm{mmol} / \mathrm{L} \mathrm{ABTS}$ and $2.45 \mathrm{mmol} / \mathrm{L}$ potassium persulphate solutions. The working solution was prepared by diluting the stock solution with phosphate buffer solution (75 mmol/L, $\mathrm{pH}=7.2$ ) to an absorbance of $0.700 \pm 0.003$ at $734 \mathrm{~nm}$. Then, $2 \mathrm{~mL}$ of $\mathrm{ABTS}^{\bullet}{ }^{*}$ working solution were mixed with $0.1 \mathrm{~mL}$ sample. The absorbance was read at $734 \mathrm{~nm}$, $15 \mathrm{~min}$ after the reaction started. A standard curve was obtained by using Trolox $(0.005-0.05 \mathrm{mmol} / \mathrm{L})$ and results were expressed as $\mu \mathrm{mol} / \mathrm{L} \mathrm{TE}$.

ORAC (oxygen radical absorbance capacity) assay was conducted as described by Zulueta et al. [2009b] with minor modification which was modification of value of $\mathrm{C}_{\text {Trolox }}$ used in the following equation. Briefly, $0.25 \mathrm{~mL}$ of samples were mixed with $1.5 \mathrm{~mL}$ of $4 \mathrm{nmol} / \mathrm{L}$ fluorescein. This solution mixture was incubated at $37^{\circ} \mathrm{C}$ for $15 \mathrm{~min}$. After this period, $0.25 \mathrm{~mL}$ of $160 \mathrm{mmol} / \mathrm{L} \mathrm{AAPH} \mathrm{(2,2'-azobis(2-methylprop-}$ ionamidine)) was added to start reaction and fluorescence intensity was read immediately until the fluorescence intensity became less than $5 \%$ of the value of the initial reading. Subsequent readings were made every $2 \mathrm{~min}$ at the wavelengths of excitation and emission of 493 and $515 \mathrm{~nm}$, respectively. ORAC values were calculated by applying the following equation and the results were expressed as $\mu \mathrm{mol} / \mathrm{L} \mathrm{TE}$.

$$
\begin{gathered}
\text { ORAC }=\left[\mathrm{C}_{\text {Trolox }} \mathrm{x}\left(\mathrm{AUC}_{\text {Sample }}-\mathrm{AUC}_{\text {Blank }}\right) \mathrm{xk}\right] / \\
/\left(\mathrm{AUC}_{\text {Trolox }}-\mathrm{AUC}_{\text {Blank }}\right)
\end{gathered}
$$

where $\mathrm{C}_{\text {Trolox }}$ is the Trolox concentration $(0.3 \mu \mathrm{mol} / \mathrm{L})$, $\mathrm{k}$ is the sample dilution factor, and AUC is the area under the fluorescence decay curve. The area under the curve (AUC) was calculated according to the following equation:

$$
\mathrm{AUC}=\left(\mathrm{f}_{0}-\mathrm{f}_{\mathrm{n}+2}\right)+2\left(\mathrm{f}_{2}+\mathrm{f}_{4}+\mathrm{f}_{6}+\ldots \ldots \ldots+\mathrm{f}_{\mathrm{n}+2}\right)
$$

where $\mathrm{f}_{0}$ is the initial relative fluorescence intensity at $0 \mathrm{~min}$ and $\mathrm{f}_{2}, \mathrm{f}_{4}, \mathrm{f}_{6, \ldots \ldots \ldots} \mathrm{f}_{\mathrm{n}+2}$ are the relative fluorescence intensities at times, 2, 4, 6, and $\mathrm{n}+2 \mathrm{~min}$.

\section{Determination of total phenolic content}

Total phenolic contents (TPC) were determined according to the Fu et al. [2011] with slight modification which was modification of added amount of Folin-Ciocalteu reagent. Briefly, $0.5 \mathrm{~mL}$ of the sample was added into $1.0 \mathrm{~mL}$ of $1: 10$ diluted Folin-Ciocalteu reagent. After $4 \mathrm{~min}, 1.0 \mathrm{~mL}$ of saturated so- dium carbonate solution (about $75 \mathrm{~g} / \mathrm{L}$ ) was added. This solution mixture was then incubated for $2 \mathrm{~h}$ at room temperature. After incubation, the absorbance was measured at $760 \mathrm{~nm}$. A standard curve was obtained by using gallic acid (0.02$-0.40 \mathrm{mmol} / \mathrm{L}$ ) and results were expressed as milligram of gallic acid equivalent per one liter of raki sample (mg GAE/L).

\section{Limit of detection (LOD) and limit of quantification (LOQ)}

LOD and LOQ were evaluated on the standard deviation of the response of the blank and the slope using the ratio $3.3 \sigma / \mathrm{S}$ and $10 \sigma / \mathrm{S}$, respectively, where $\sigma$ is the standard deviation of the response of 10 blank samples and $\mathrm{S}$ is the slope of the calibration curve of the analyte.

\section{Statistical analysis}

All values were analysed by GraphPad Prism 5 and Microsoft Excel Software. Three independent aliquots of the sample were measured and all measurements were replicated three times for each sample. The data were expressed as means \pm standard deviation (SD). The one-way ANOVA and Bonferroni tests were used to determine differences among means and the differences were considered as significant with $\mathrm{p}<0.05$. Pearson's correlation analysis was performed between the antioxidant capacity and TPC.

\section{RESULTS AND DISCUSSION}

The antioxidant capacity of raki samples which was determined by CUPRAC, DPPH, TEAC and ORAC assays are shown in Table 1. The results pointed out that all raki samples displayed antioxidant properties. The highest antioxidant capacity values were obtained from sample F (dried type grapes), followed by E (dried type grapes), in CUPRAC, TEAC and ORAC assays. For the DPPH assay, E showed the highest antioxidant capacity, followed by A (dried type grapes). Sample G (fresh type grapes) exhibited the lowest radical scavenging activity in DPPH and TEAC assays. In terms of the ORAC and CUPRAC assays, B (dried type grapes) and $\mathrm{C}$ (fresh type grapes) displayed the lowest results, respectively. To the best of our knowledge, there has been no study that evaluated antioxidant capacity of raki. However, Pellegrini et al. [2003] investigated some alcoholic drinks TEAC values. They reported 1290, 40 and $1040 \mu \mathrm{mol} / \mathrm{L} \mathrm{TE}$ for cognac, rum and beer, respectively. Mitić et al. [2013] reported the DPPH and TEAC values of beers ranged from 260-830 $\mu \mathrm{mol} / \mathrm{L} \mathrm{TE}$ and 140-350 $\mu \mathrm{mol} / \mathrm{L} \mathrm{TE}$, respectively. The results obtained from this study are in agreement with data found for the other alcoholic drinks.

Studies have indicated that the major constituents of aniseed are anethole, estragole, eugenol, umbelliferon, coumarins [Gülçın et al., 2003]. Grapes are rich in phenolic compounds such as gallic acid, caffeic acid, chlorogenic acid, kaempferol, quercetin [dos Lima et al., 2014]. These phenolic compounds have been considered to have high antioxidant activity. Antioxidant properties of raki samples may be attributed to these useful natural compounds.

TPC of raki samples were evaluated by using Folin Ciocalteu reagent. In general, TPC results of samples showed 
TABLE 1. Antioxidant capacities ( $\mu \mathrm{mol} / \mathrm{L} \mathrm{TE})$ and TPC (mg GAE/L) of raki samples.

\begin{tabular}{l|c|c|c|c|c}
\hline \multirow{2}{*}{ Samples } & \multicolumn{5}{c}{ Methods } \\
\cline { 2 - 6 } & CUPRAC & DPPH & TEAC & ORAC & TPC \\
\hline A & $100.43 \pm 5.17$ & $59.00 \pm 3.61$ & $121.35 \pm 9.46$ & $207.34 \pm 6.52$ & $8.31 \pm 0.40$ \\
B & $97.20 \pm 3.11$ & $55.37 \pm 4.51$ & $98.51 \pm 4.43$ & $105.96 \pm 5.68$ & $5.83 \pm 0.30$ \\
C & $87.33 \pm 2.29$ & $46.13 \pm 4.50$ & $107.66 \pm 5.98$ & $176.80 \pm 9.08$ & $5.42 \pm 0.35$ \\
D & $122.62 \pm 2.36$ & $49.30 \pm 5.11$ & $114.86 \pm 7.45$ & $177.48 \pm 4.47$ & $7.42 \pm 0.21$ \\
E & $132.79 \pm 4.03$ & $61.70 \pm 1.13$ & $142.34 \pm 9.60$ & $211.12 \pm 11.19$ & $8.48 \pm 0.12$ \\
F & $141.84 \pm 9.18$ & $58.62 \pm 2.63$ & $152.97 \pm 7.37$ & $232.99 \pm 8.92$ & $8.82 \pm 0.24$ \\
G & $109.60 \pm 8.91$ & $43.52 \pm 1.96$ & $95.95 \pm 6.84$ & $181.82 \pm 9.03$ & $6.93 \pm 0.50$ \\
\hline
\end{tabular}

Data are expressed as means \pm SD. $p<0.05$. Dried type grapes (A, B, E, F), fresh type grapes (C, G), fresh-dried type grapes (D).

similar trend with antioxidant capacity assays in this study, where sample $\mathrm{F}$ (dried type grapes) exhibited the highest TPC followed by E, A, D, G, B, (A, B, E; dried type grapes, $\mathrm{D}$; fresh-dried, $\mathrm{G}$; fresh) while sample $\mathrm{C}$ (fresh type grapes) demonstrated the lowest TPC (Table 1).

Antioxidant capacity and TPC values of samples were found statistically different by ANOVA-One way test for all assays $(\mathrm{p}<0.05)$. In general, raki samples made from dried type grapes revealed higher antioxidant capacity and TPC than those made from fresh type grapes in this study. Bonferroni test was performed to evaluate differences between values of samples. Results are seen in Table 2. Based on this post-hoc test results, while statistical differences between four different sample pairs were found significant, two different sample pairs showed no statistical differences for all assays. Generally, this test showed significant differences between raki samples made from dried type grapes and those made from fresh type grapes. This confirmed the higher antioxidant capacity values of raki samples made from dried type grapes.

The influence of the method on antioxidant capacity was evaluated and the corresponding descriptive statistic data are presented in Table 3. The mean antioxidant capacity values of raki samples were in the order of: ORAC $>$ TEAC $>$ CUPRAC $>$ DPPH. Tafulo et al. [2010] investigated the antioxidant capacity of different beer samples with the aid of TRAP (total radical trapping antioxidant parameter), TEAC, DPPH, FRAP (ferric-ion reducing antioxidant parameter), CUPRAC and ORAC assays. Like results found in the present study, they obtained the highest antioxidant capacity value by ORAC assay, while DPPH assay displayed the lowest value. As can be seen from Table 3, the mean values of CUPRAC and TEAC methods are very close to each other, 113.12 and $119.09 \mu \mathrm{mol} / \mathrm{L}$ TE, respectively. This could be due to the fact that CUPRAC and TEAC are similar antioxidant capacity assays with close reduction potentials.

The correlations between antioxidant capacity and TPC of samples are evaluated in the present study. The correlation of TPC of samples with their CUPRAC, TEAC and ORAC (0.8071, 0.8196 and 0.7998 , respectively) antioxidant capacities were found statistically significant. These significant correlations indicated that phenolic compounds could be one
TABLE 2. Bonferroni post-hoc test results.

\begin{tabular}{|c|c|c|c|c|c|}
\hline Samples & CUPRAC & DPPH & TEAC & ORAC & TPC \\
\hline $\mathrm{A} v s \mathrm{~B}$ & ns & ns & $*$ & * & * \\
\hline $\mathrm{A} v s \mathrm{C}$ & ns & $*$ & ns & $*$ & * \\
\hline $\mathrm{A} v s \mathrm{D}$ & * & ns & ns & * & ns \\
\hline $\mathrm{A} v s \mathrm{E}$ & $*$ & ns & ns & ns & ns \\
\hline $\mathrm{A} v s \mathrm{~F}$ & $*$ & ns & * & * & ns \\
\hline $\mathrm{A} v s \mathrm{G}$ & ns & $*$ & * & * & * \\
\hline $\mathrm{B} v_{s} \mathrm{C}$ & ns & ns & ns & * & ns \\
\hline $\mathrm{B} v s \mathrm{D}$ & * & ns & ns & * & $*$ \\
\hline $\mathrm{B} v s \mathrm{E}$ & * & ns & * & $*$ & $*$ \\
\hline $\mathrm{B} v s \mathrm{~F}$ & $*$ & ns & $*$ & * & * \\
\hline $\mathrm{B} v s \mathrm{G}$ & ns & $*$ & ns & $*$ & $*$ \\
\hline C vs D & $*$ & ns & ns & ns & $*$ \\
\hline $\mathrm{C} v s \mathrm{E}$ & $*$ & $*$ & * & $*$ & $*$ \\
\hline $\mathrm{C} v s \mathrm{~F}$ & $*$ & $*$ & $*$ & * & $*$ \\
\hline $\mathrm{C} v s \mathrm{G}$ & $*$ & ns & ns & ns & $*$ \\
\hline $\mathrm{D} v s \mathrm{E}$ & ns & $*$ & * & $*$ & $*$ \\
\hline $\mathrm{D} v s \mathrm{~F}$ & * & ns & $*$ & * & $*$ \\
\hline $\mathrm{D} v s \mathrm{G}$ & ns & ns & ns & ns & ns \\
\hline $\mathrm{E} v s \mathrm{~F}$ & ns & ns & ns & ns & ns \\
\hline $\mathrm{E} v s \mathrm{G}$ & * & $*$ & $*$ & $*$ & $*$ \\
\hline $\mathrm{F} v s \mathrm{G}$ & $*$ & $*$ & $*$ & $*$ & * \\
\hline
\end{tabular}

*Indicates significant differences at $\mathrm{p}<0.05$. ns; not significant. Dried type grapes (A, B, E, F), fresh type grapes (C, G), fresh-dried type grapes (D).

of the main components responsible for free radical scavenging ability of raki samples. However, considering the results for DPPH, a significant correlations between TPC and DPPH (0.6643) could not be found. The DPPH assay is technically simple, but some disadvantages limit its applications. 
TABLE 3. Descriptive statistics of antioxidant capacity ( $\mu \mathrm{mol} / \mathrm{L} \mathrm{TE})$ and TPC (mg GAE/L) assays.

\begin{tabular}{|c|c|c|c|c|c|c|c|c|c|}
\hline \multirow{2}{*}{ Method } & \multirow{2}{*}{$\begin{array}{l}\text { Number } \\
\text { of samples }\end{array}$} & \multirow{2}{*}{ Mean } & \multirow{2}{*}{ SD } & \multicolumn{2}{|c|}{ Confidence interval 95\% } & \multirow{2}{*}{ Max. } & \multirow{2}{*}{ Min. } & \multirow{2}{*}{ LOD } & \multirow{2}{*}{ LOQ } \\
\hline & & & & Upper limit & Lower limit & & & & \\
\hline CUPRAC & 7 & 113.12 & 19.97 & 127.94 & 98.30 & 141.84 & 87.33 & 5.52 & 16.73 \\
\hline DPPH & 7 & 53.38 & 7.06 & 58.62 & 48.14 & 61.70 & 43.52 & 5.48 & 16.61 \\
\hline TEAC & 7 & 119.09 & 21.61 & 135.13 & 103.06 & 152.97 & 95.95 & 1.33 & 4.05 \\
\hline ORAC & 7 & 184.79 & 40.55 & 214.89 & 154.69 & 232.99 & 105.96 & 0.03 & 0.09 \\
\hline TPC & 7 & 7.32 & 1.33 & 8.30 & 6.34 & 8.82 & 6.34 & 0.63 & 1.92 \\
\hline
\end{tabular}

DPPH is a long lived nitrogen radical. Many antioxidants may react slowly or may even be inert to this radical [Huang et al., 2005; Bernaert et al., 2012]. This could explain why results obtained by DPPH assay were found non-significant with TPC results. Many studies have shown significant correlations between the results after analysing antioxidant capacity and TPC of various food samples. Bernaert et al. [2012] examined antioxidant capacity of leek extracts. They also reported significant correlation coefficient between ORAC and TPC, but in the same study they also found no statistically significant correlation between the results of DPPH and TPC assays. Lu et al. [2011] and Song et al. [2010] detected significant correlation between TPC and TEAC. Apak et al. [2006] investigated the antioxidant capacity of different medicinal and food herbs. They showed that the TPC results of samples correlated significant with those of CUPRAC and TEAC.

\section{CONCLUSIONS}

The finding of this study indicated that raki has antioxidant properties. However, antioxidant capacity values of raki samples showed variations based on method used in this investigation. The highest results were obtained by using ORAC and the lowest results were obtained by using DPPH assay. Raki samples from different brands also showed different antioxidant capacity and TPC values. In general, correlations between antioxidant capacity assays and TPC were found statistically significant.

\section{ACKNOWLEDGEMENTS}

The author wish to thank Ege University Faculty of Pharmacy Pharmaceutical Sciences Research Centre (FABAL).

\section{REFERENCES}

1. Anli R.E., Bayram M., Traditional aniseed-flavored spirit drinks. Food Rev. Int., 2010, 26, 246-269.

2. Apak R., Güçlü K., Özyürek M., Karademir S.E., Erçağ E., The cupric ion reducing antioxidant capacity (CUPRAC) and polyphenolic content of some herbal teas. Int. J. Food Sci. Nutr., 2006, 57, 292-304.

3. Apak R., Güçlü K., Özyürek M., Çelik S.E., Mechanism of antioxidant capacity assays and the CUPRAC (cupric ion reducing antioxidant capacity) assay. Microchim. Acta, 2008, 160, 413-419.
4. Bernaert N., de Paepe D., Bouten C., de Clercq H., Stewart D., Bockstaele E.V., de Loose M., Droogenbroeck B.V., Antioxidant capacity, total phenolic and ascorbate content as a function of the genetic diversity of leek (Allium ampeloprasum var. porrum). Food Chem., 2012, 134, 669-677.

5. dos Lima M.S., de Silani I.S.V., Toaldo I.M., Correa L.C., Biasoto A.C.T., Pereira G.E., Bordignon-Luiz M.T., Ninow J.L., Phenolic compounds, organic acids and antioxidant activity of grape juices produced from new Brazilian varieties planted in the Northeast Region of Brazil. Food Chem., 2014, 161, 94-103.

6. Fu L., Xu B.T., Xu X. R., Gan R.Y., Zhang Y., Xia E.Q., Li H.B., Antioxidant capacities and total phenolic contents of 62 fruits. Food Chem., 2011, 129, 345-350.

7. Gülçın İ., Oktay M., Kıreçcı E., Küfrevıoğlu Ö.İ., Screening of antioxidant and antimicrobial activities of anise (Pimpinella anisum L.) seed extracts. Food Chem., 2003, 83, 371-382.

8. Huang D., Ou B., Prior R.L., The chemistry behind antioxidant capacity assays. J. Agric. Food Chem., 2005, 53, 1841-1856.

9. Li J.W., Ding S.D., Ding X.L., Comparison of antioxidant capacities of extracts from five cultivars of Chinese jujube. Process Biochem., 2005, 40, 3607-3613.

10. Li P.M., Du G.R., Ma F.W., Phenolics concentration and antioxidant capacity of different fruit tissues of astringent versus nonastringent persimmons. Sci. Hort., 2011, 129, 710-714.

11. Lu M., Yuan B., Zeng M., Chen J., Antioxidant capacity and major phenolic compounds of spices commonly consumed in China. Food Res. Int., 2011, 44, 530-536.

12. Meng J., Fang Y., Zhang A., Chen S., Xu T., Ren Z., Han G., Liu J., Li H., Zhang Z., Wang H., Phenolic content and antioxidant capacity of Chinese raisins produced in Xinjiang Province. Food Res. Int., 2011, 44, 2830-2836.

13. Mitić S.S., Paunović D.D., Pavlović A.N., Tošić S.B., Stojković M.B., Mitić M.N., Phenolic profiles and total antioxidant capacity of marketed beers in Serbia. Int. J. Food Prop., 2014, 17, 908-922.

14. Özyürek M., Güçlü K., Apak R., The main and modified CUPRAC methods of antioxidant measurement. Trends Anal. Chem., 2011, 30, 652-664.

15. Pellegrini N., Serafini M., Colombi B., Rio D.D., Salvatore S., Bianchi M., Brighenti F., Total antioxidant capacity of plant foods, beverages and oils consumed in Italy assessed by three different in vitro assays. J. Nutr., 2003, 133, 2812-2819.

16. Re R., Pellegrini N., Proteggente A., Pannala A., Yang M., RiceEvans C., Antioxidant activity applying an improved ABTS radical cation decolourisation assay. Free Radic. Biol. Med., 1999, 26, 1231-1237. 
17. Schwarz M., Rodríguez M., Martínez C., Bosquet V., Guillén D., Barroso C.G., Antioxidant activity of Brandy de Jerez and other aged distillates, and correlation with their polyphenolic content. Food Chem., 2009, 116, 29-33.

18. Song F.L., Gan R.Y., Zhang Y., Xiao Q., Kuang L., Li H.B., Total phenolic contents and antioxidant capacities of selected Chinese medicinal plants. Int. J. Mol. Sci., 2010, 11, 2362-2372.

19. Tafulo P.A.R., Queiros R.B., Delerue-Matos C.M., Sales M.G.F., Control and comparison of the antioxidant capacity of beers. Food Res. Int., 2010, 43, 1702-1709.

20. Yucesoy D., Ozen B., Authentication of a Turkish traditional aniseed flavoured distilled spirit, raki. Food Chem., 2013, 141, 1461-1465.
21. Zulueta A., Esteve M.J., Frasquet I., Frígola A., Vitamin C, vitamin $\mathrm{A}$, phenolic compounds and total antioxidant capacity of new fruit juice and skim milk mixture beverages marketed in Spain. Food Chem., 2007, 103, 1365-1374.

22. Zulueta A., Esteve M.J., Frígola A., ORAC and TEAC assays comparison to measure the antioxidant capacity of food products. Food Chem., 2009a, 114, 310-316.

23. Zulueta A., Maurizi A., Frígola A., Esteve M.J., Coli R., Burini G., Antioxidant capacity of cow milk, whey and deproteinized milk. Int. Dairy J., 2009b, 19, 380-385.

Submitted: 10 December 2014. Revised: 1 April 2015. Accepted: 27 April 2015. Published on-line: 1 March 2016. 